\title{
PHIL Migration to Internal Carotid Artery and Middle Cerebral Artery: Late Mechanical Retrieval with Solitaire AB Stent
}

\author{
Giancarlo Saal-Zapata ${ }^{1 \odot ~ W a l t e r ~ D u r a n d ~}{ }^{1}$ Ricardo Vallejos ${ }^{1}$ Dante Valer ${ }^{1} \quad$ Jesús Flores ${ }^{1}$ \\ Rodolfo Rodriguez ${ }^{1}$
}

${ }^{1}$ Department of Neurosurgery, Endovascular Neurosurgery Service, Hospital Nacional Guillermo Almenara Irigoyen-EsSalud, La Victoria, Lima, Peru

J Clin Interv Radiol ISVIR:2021;5:51-54

\begin{abstract}
Address for correspondence Giancarlo Saal-Zapata, MD, Department of Neurosurgery, Endovascular Neurosurgery Service. Hospital Nacional Guillermo Almenara Irigoyen-EsSalud, Grau Avenue 800, La Victoria, Lima 13, Peru (e-mail: gian_carlo1987@hotmail.com; gsaal1987@gmail.com).
\end{abstract}

\begin{abstract}
Keywords

- PHIL

- microcatheter

- arteriovenous

malformation

Precipitating hydrophobic injecting liquid (PHIL) is a novel embolic agent used in the endovascular treatment of arteriovenous malformations (AVM) and arteriovenous fistulas. Complications can occur during embolization with migration of the liquid embolic material to normal vasculature. In these cases, use of a stent retriever is an option for removal of the plug. Herein, we present the case of a patient who presented with a right occipital hemorrhage due to a ruptured occipital AVM fed by the calcarine and parieto-occipital arteries with venous drainage to the straight sinus. Embolization via the anterior circulation through the right posterior communicating artery was attempted but PHIL migrated to right internal carotid artery and middle cerebral artery due to kinking and rupture of the microcatheter. The patient developed mild left hemiparesis. When this complication occurred, no stent retriever was available at our institution and 1 day later, the Solitaire $A B$ stent was used to remove the plug of PHIL successfully, with good reperfusion of the proximal and distal vessels. The patient improved after the procedure and was discharged home without deficit. Mechanical stent retrievers can be used in cases of migration of liquid embolic agents to normal vasculature with good clinical and radiological results.
\end{abstract}

\section{Introduction}

Precipitating hydrophobic injecting liquid (PHIL; Microvention) is a nonadhesive dimethyl sulfoxide-based liquid embolic agent with iodine for radiopacity, unlike Onyx (Medtronic Neurovascular), which is a compound of tantalum for radiopacity. ${ }^{1}$ It is widely used for the treatment of arteriovenous malformations (AVM) and arteriovenous fistulas (AVF). PHIL advantages are: less angionecrosis, less computed tomography (CT) and magnetic resonance imaging artifacts, faster plug formation, does not require shaking prior to injection, and is easier to use.,3 In our experience, wealsonoted that PHILtends less to reflux to parent artery.

We present the case of a successful retraction of the liquid embolic agent PHIL from the anterior circulation using the Solitaire $A B$ stent (eV3) 1 day after this complication took place. There are few cases reports in the literature that describe the off-label use of stent retrievers for the removal of liquid embolic agents. To our knowledge, this is the first case where a Solitaire $A B$ stent is used for retrieving a migrated plug of PHIL from the internal carotid artery (ICA) and middle carotid artery (MCA). published online

July 21,2020
DOI https://doi.org/

10.1055/s-0040-1710152 ISSN 2457-0214. (c) 2020. Indian Society of Vascular and Interventional Radiology. This is an open access article published by Thieme under the terms of the Creative Commons Attribution-NonDerivative-NonCommercial-License, permitting copying and reproduction so long as the original work is given appropriate credit. Contents may not be used for commercial purposes, or adapted, remixed, transformed or built upon. (https://creativecommons.org/licenses/by-nc-nd/4.0/).

Thieme Medical and Scientific Publishers Pvt. Ltd. A-12, 2nd Floor, Sector 2, Noida-201301 UP, India 


\section{Case Description}

A 38-year-old man, previously healthy, presented to the emergency with headache and nausea associated with vomiting. Brain CT scan revealed a right occipital hemorrhage and was referred to our institution for complementary studies. Digital subtraction angiography (DSA) revealed a right ruptured occipital AVM with feeding vessels from the right calcarine and parieto-occipital arteries and venous drainage to the straight sinus, being catalogued as grade III and II according to SpetzlerMartin and Buffalo classification, respectively ( - Fig. 1). The ipsilateral posterior communicating artery (PComA) was dominant. We decided to perform the embolization of the AVM with PHIL via the anterior circulation through the right PComA because the trajectory to the AVM nidus would be safer compared with the posterior circulation approach.

The procedure was held in the angio-suite using the biplane flat panel digital subtraction unit (Allura Xper FD20/10, Phillips Healthcare). Under general anesthesia, a 6-French Envoy guiding catheter (Cordis Neurovascular) was placed in the C1 segment of the right ICA. The Sonic (Balt Extrusion) detachable tip microcatheter over $n$ Hybrid 007D micro guidewire (Balt Extrusion) was navigated through the right PComA and located proximal to the AVM nidus. A super-selective injection confirmed the adequate position of the microcatheter, but it was kinked with a loop originating at the tip of the guide catheter and proximally to the AVM nidus ( - Fig. 2A, B). PHIL injection started but penetration to the nidus was not observed. A control angiogram showed that PHIL migrated to the ICA bifurcation and M1 segment of the MCA with partial occlusion of these vessels ( - Fig. 2C-F).

At the time this complication occurred, no stent was available at our institution. Heparin with dual antiplatelet therapy was initiated and the procedure was suspended. A CT scan
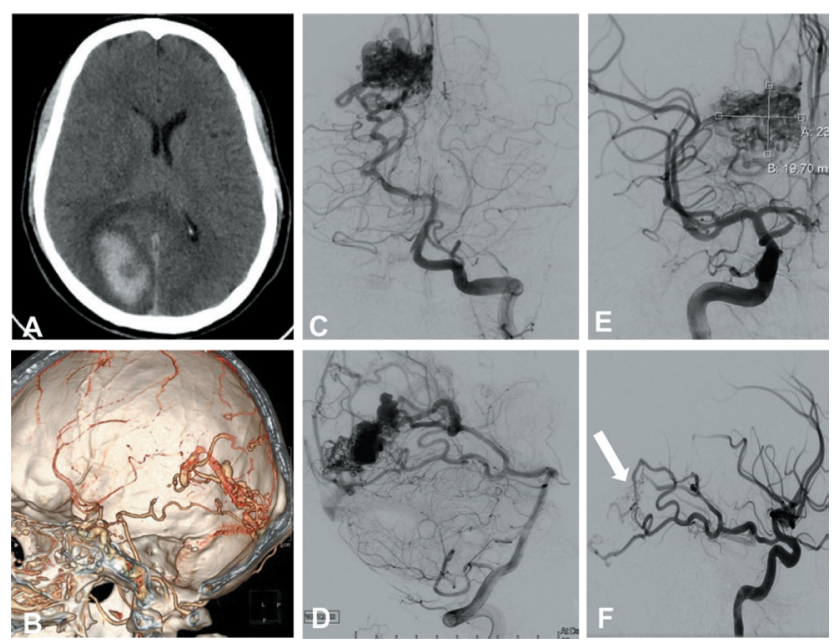

Fig. 1 Pre-embolization arteriovenous malformation (AVM) characteristics. (A) Brain computed tomography (CT) shows right occipital hemorrhage. (B) CTA shows the AVM nidus. (C, D) Digital subtraction angiography (DSA) of the left vertebral artery in anterior-posterior and lateral views showing a right occipital AVM with feeding arteries from the calcarine and parieto-occipital arteries with venous drainage to the straight sinus. (E, F) DSA of the right internal carotid artery showing the AVM filling from the ipsilateral posterior communicating artery; the arrow shows the AVM nidus in the lateral view. revealed the migration of PHIL to the MCA territory, but no signs of ischemia or infarct were found ( - Fig. 2G, H). The patient had a National Institutes of Health Stroke Scale score of three points, with mild hemiparesis.

The angiogram performed the day after the initial procedure showed persistent plug of PHIL in the vessels. We attempted removal of the plug with a stent retriever. A $6 \mathrm{~F}$ Envoy guide catheter was placed in the right ICA and the Headway 27 microcatheter (Microvention) over a Transend 14 microwire (Stryker) was navigated across the occluded arteries. Once in the correct position, the $6 \times 20 \mathrm{~mm}$ Solitaire $\mathrm{AB}$ stent was deployed. The plug could be removed completely from the ICA and partially from M1 ( - Fig. 3A, B).

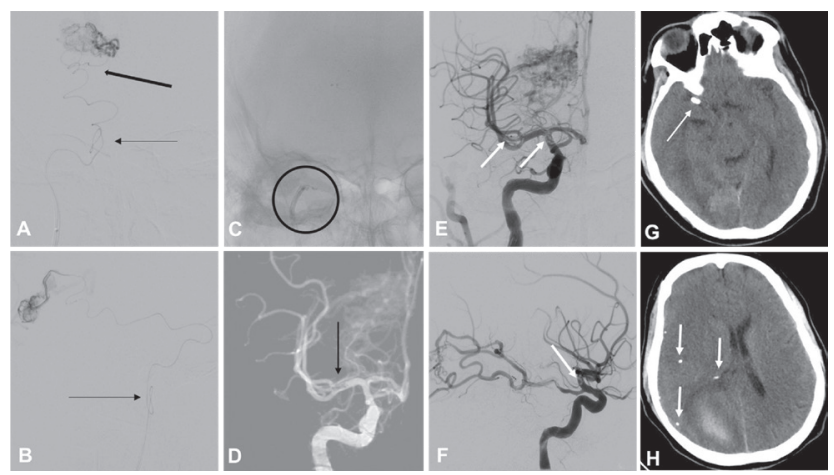

Fig. 2 (A, B) Super-selective angiogram of the arteriovenous malformation nidus via the anterior circulation through the right posterior communicating artery; the arrows show the kinking of the microcatheter. (C) Precipitating hydrophobic injecting liquid (PHIL) migration to the internal carotid artery (ICA) while injecting. (D) Road mapping shows the presence of the plug (arrow) in the middle cerebral artery (MCA). (E, F) Digital subtraction angiography (DSA) showing PHIL migration to ICA and MCA (arrows). (G, H) Brain computed tomography scan shows the presence of PHIL in the ICA and migration to the MCA territory (multiple arrows).
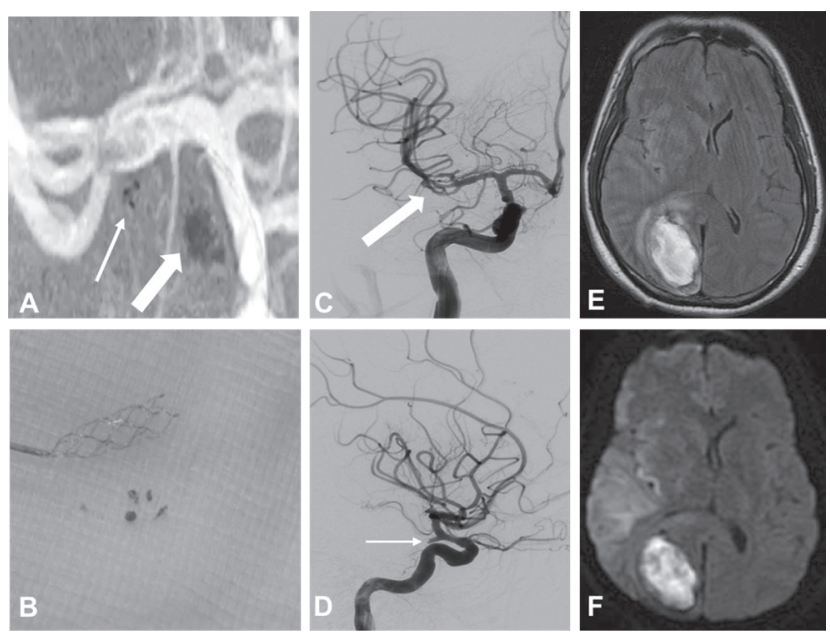

Fig. 3 (A) Road mapping showing removal of precipitating hydrophobic injecting liquid (PHIL) plug with Solitaire $A B$ stent (thin arrow $=$ stent deployed, big arrow $=$ PHIL been removed). $($ B $)$ Solitaire $A B$ stent containing PHIL residues. (C, D) Digital subtraction angiography after PHIL plug retrieval with complete reperfusion of the middle cerebral artery (big arrow, $\mathbf{C}$ ) and absence of posterior communicating artery (thin arrow, D) due to PHIL migration. (E, F) Magnetic resonance imaging after PHIL retrieval shows signs of infarct. 
The second pass removed the plug from the M1 and the control angiogram showed total reperfusion of the MCA (-Fig. 3C), but migration of fragments occluded the ipsilateral PComA (-Fig. 3D). Aspiration was not attempted to remove the plug within the PComA. A postoperative magnetic resonance showed an infarct in the MCA territory (-Fig. 3E, F). The patient improved clinically with a post-procedure modified Rankin Scale score of 0 . He was discharged home without any neurological deficits. The patient later underwent total embolization of the AVM through the left vertebral artery without complications (-Fig. 4).

\section{Discussion}

Liquid embolic agents such as N-butyl-2 cyanoacrylate, Onyx, and PHIL are widely used for the endovascular treatment of AVMs and AVFs.

Vessel perforation, migration of embolic materials, and reflux to parent artery are possible complications while treating these vascular entities. ${ }^{4}$

Many studies report good clinical and radiological results with the use of stent retrievers, ${ }^{5}$ especially with the Solitaire stent. Removal of embolic plugs from the normal vasculature due to technical complications is not the main indication and should be used in selected cases.

In developing countries, the availability of endovascular devices is sometimes limited because of their high cost. Therefore, endovascular treatments are not performed frequently in most of the hospitals. In Peru, fortunately we have EsSalud (Peruvian Social Security System) that encompasses highly specialized centers for the treatment of vascular pathology, providing endovascular materials for the treatment of complex cases.

We searched in PubMed for similar cases in which a mechanical thrombectomy device was used to retrieve liquid embolic agents from the arteries or veins and found few

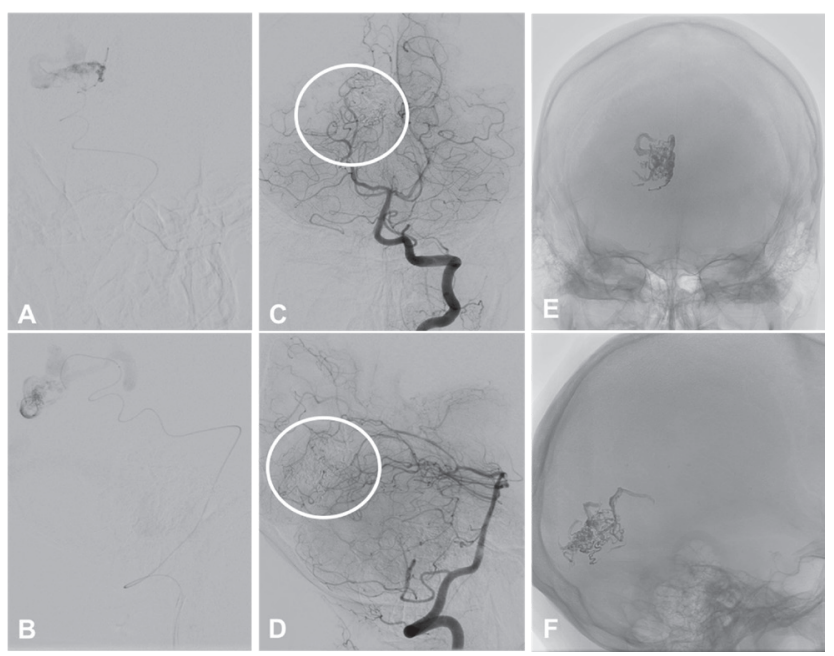

Fig. 4 Embolization of the arteriovenous malformation (AVM) through the left vertebral artery: (A, B) Super-selective injection showing the microcatheter in the AVM nidus. (C, D) Control angiogram showing the total obliteration of the AVM nidus; the circle depicts the presence of the cast. (E, F) Presence of precipitating hydrophobic injecting liquid cast. cases. Merci (Concentric Medical) has been used for retrieving Onyx from the vertebrobasilar system.,

Michael et al in 2009 reported the first case of Onyx withdrawal using the Merci L6 retriever device in a patient in whom Onyx refluxed to the vertebrobasilar junction while performing the embolization of a posterior fossa AVM. ${ }^{7}$ The complete removal of the substance was achieved and the patient improved clinically.

In 2015, Senturk ${ }^{6}$ reported another case of Onyx retrieval from the vertebrobasilar junction due to microcatheter rupture. In this case, the Merci retriever device was used with partial removal of the plug. In the same year, Fahed et $\mathrm{al}^{8}$ reported the use of Solitaire FR for retrieving N-butyl-2 cyanoacrylate from the venous system. Moreover, Li et al reported the use of Solitaire $A B$ to retrieve Onyx from the basilar artery. ${ }^{9}$ Thus, stent retrievers have been used to remove liquid embolic agents from the normal vasculature..$^{9,10}$

In this case, the AVM was catheterized via the anterior circulation; the guide catheter was placed in an adequate position, but the microcatheter kinked and formed two loops in its trajectory. PHIL administration did not reach the AVM nidus and escaped through the kink at the tip of the guide catheter. We hypothesized that the kinking of the microcatheter leads to its rupture in the proximal loop with migration of PHIL. Technically, a previous super-selective angiogram was performed without forcing the injection. The microcatheter was found broken after it was removed.

After analyzing the case, we decided to use a stent retriever. We have to consider that the removal of the plug was performed many hours after this complication took place. This occurred because of a lack of the stent at that moment; moreover, the vessel filling was adequate and let us plan the solution for this complication. This is an important issue to consider as in all the previous reported cases removal of the plug was performed immediately.

This is the first case reporting the retrieval of PHIL in a delayed fashion from the ICA and MCA in the same procedure, using a stent retriever.

The key message of this case is that: the use of stent retrievers for large vessel occlusion due to liquid embolic agent migration in a delayed fashion is a feasible technique; however, it should be performed in selected cases.

\section{Conclusion}

The use of the Solitaire stent retriever is a safe and feasible option for the retraction of migrated liquid embolic materials.

\section{Conflicts of Interest}

There are no conflicts of interest.

\section{References}

1 Vollherbst DF, Sommer CM, Ulfert C, Pfaff J, Bendszus M, Möhlenbruch MA. Liquid embolic agents for endovascular embolization: Evaluation of an established (Onyx) and a novel (PHIL) embolic agent in an in vitro AVM model. AJNR Am J Neuroradiol 2017;38(7):1377-1382 
2 Koçer N, Hanımoğlu H, Batur ş, et al. Preliminary experience with precipitating hydrophobic injectable liquid in brain arteriovenous malformations. Diagn Interv Radiol 2016;22(2):184-189

3 Vollherbst DF, Otto R, Do T, et al. Imaging artifacts of Onyx and PHIL on conventional CT, cone-beam CT and MRI in an animal model. Interv Neuroradiol 2018;24(6):693-701

4 Poncyljusz W, Sawicki M, Lubkowska K, Rać M. Early outcomes and periprocedural complications of transarterial embolization of brain arteriovenous malformations with Onyx ${ }^{\circledR}$. Neurol Neurochir Pol 2017;51(4):277-285

5 Matsumaru Y, Ishikawa E, Yamamoto T, Matsumura A. Recent trends in neuro-endovascular treatment for acute ischemic stroke, cerebral aneurysms, carotid stenosis, and brain arteriovenous malformations. Neurol Med Chir (Tokyo) 2017;57(6):253-260

6 Senturk C. Mechanical removal of migrated onyx due to microcatheter rupture during AVM embolization: a technical case report. Cardiovasc Intervent Radiol 2015;38(6):1654-1657
7 Michael SG, Swarnkar AS, Latorre JG, Ramachandran TS, Lodi YM. Revascularization of Onyx induced intra-operative occlusion of vertebro-basilar artery using the Merci device. Neurocrit Care 2010;12(2):269-271

8 Fahed R, Clarençon F, Sourour NA, et al. Rescue N-butyl-2 cyanoacrylate embolectomy using a Solitaire FR device after venous glue migration during arteriovenous malformation embolization: technical note. J Neurosurg 2016;125(1):173-176

9 Li M, Guo Q, Liu W, Yan Z. Liquid embolic onyx reflux to basilar artery retrieved by solitaire ab stent in the treatment of arteriovenous malformations. J Vasc Interv Radiol 2015;26(6):927-929

10 Gungor D, Oğuz Ş, Dinc H. Mechanical removal of a refluxed Onyx piece from the middle cerebral artery using the Solitaire stent: technical report. Interv Neuroradiol 2017;23(3): 293-296 